\title{
Development of a Dynamic Model of Quality Performance in Thai Construction Industry
}

\author{
${\text { Thanwadee } \text { Chinda }^{1 *}, K \text {. Sittimak }}^{2}, N$. Thalaengkij $^{2}, P$. Chaiyapong $^{2}, P$. Sornpasit ${ }^{2}$, \\ $W$. K etnak ${ }^{2}$ \\ ${ }^{1}$ School of $\mathrm{M}$ anagement Technology, Sirindhorn International Institute of Technology, \\ Thammasat U niversity, 131 Tiwanont Rd. B angkadi M uang Pathumthani 12000 Thailand, \\ Email: thanwadee@ siit.tu.ac.th \\ ${ }^{2} \mathrm{~S}$ chool of M anagement Technology, Sirindhorn International Institute of Technology, \\ Thammasat U niversity, 131 Tiwanont Rd. B angkadi M uang Pathumthani 12000 Thailand
}

\begin{abstract}
Many quality performance tools are implemented in the construction industry to enhance the quality performance standard. This paper utilizes an ISO 9001 standard implementation to develop a dynamic model of construction quality performance in the Thai construction industry. The model consists of five sub-models, namely the Leadership, Plan, Do, Check, and Act sub-models. Five levels of performance maturity are also developed in this study to plan for long-term improvement. The simulation results reveal that a company takes seven years to proceed from level 1 to level 2 of maturity as it lacks experience and knowledge. With more experience, the company progresses through to higher maturity levels in a shorter period of time. The model achieves the perfect implementation score of 1,000 points at the end of year 23. This study results can be used to aid the construction industry in the effective planning for long-term quality improvement.
\end{abstract}

Keywords: construction industry, ISO 9001, Plan-D o-Check-Act Cycle, quality performance, system dynamics modelling

\section{Introduction}

The construction industry is dynamic in nature, and the concept of good performance in the construction industry is hard to define. Aspects of project quality are often overlooked in order to conform to the schedule and cost constraints. Many international quality standards, such as the ISO 9001 standard, Deming Cycle, and the balanced scorecard, are therefore developed as a foundation for quality management system [1]. The ISO 9001

* Corresponding author: thanwadee@ siit.tu.ac.th 
is an international standard that indicates requirements for a quality management system. It is used to demonstrate the ability of a company to consistently provide products and services that meet customer and regulatory requirements. It places a strong emphasis on product quality and continuous improvement [2]. In the South-East Asia region, the perception of ISO 9001 quality standard is good; how ever, there are several issues, such as a poor culture of continual improvement and lack of process approach utilization throughout the organization that are yet studied. Bubshait and AI-A tiq [3], for example, evaluated a contractor $\$$ quality assurance system in Saudi A rabia against the ISO 9000 standard. Pheng and Teo [4] examined the compatibility of the recently released ISO 9001: 2000 with OHSA S 18001: 1999 for the purpose of integration, and concluded that the newly introduced ISO 9001: 2000 standard serves as an opportune platform for construction firms to consider certification to OHSA S 18001: 1999 through an integration exercise.

Despite the above researches, there is a need to examine interactions among key factors affecting quality enhancement to effectively plan for construction quality improvement. Different maturity levels should also be developed so that the company can plan for construction improvement, and progress through to higher maturity levels in the long term. This paper, therefore, aims at developing a dynamic model of quality performance in the construction industry using an ISO 9001 standard. It is expected that a company can better understand causal relationships among key factors affecting construction quality, and plan for quality improvement in the long-term.

\section{Key Factors affecting Quality Performance}

\subsection{Five Key Factors Affecting quality Performance}

A ccording to [5], the ISO 9001: 2015 can be generally classified into ten factors. Gonzalez [6], however, stated that the ISO 9001:2015 management system standard clauses can be overlaid with the Plan-Do-Check-A ct (PDCA) Cycle to work in conjunction with each other. Paulova and $\mathrm{M}$ Ikva [7], on the other hand, mentioned that leadership is the key element in improving quality management. In this study, therefore, five key factors, including 1) L eadership, 2) Plan, 3) D 0, 4) Check, and 5) A ct factors are used for the dynamic model of quality performance development. They are associated with a total of 22 items extracted from a number of construction-related literature, such as Senaratne and Mayuran [8], Neyestani [9] and Shah and Pitroda [10]. They form a number of causal relationships, as shown in Figure 3. For instance, good management review leads to better and realistic quality policy and quality plan [11]. It could include the provision of proper training to enhance worker's skill. With a better skill, rework tends to reduce, and workers tend to assist each other to improve quality [12]. This, in turn, brings a better management review.

- Leadership factor: This factor consists of five associated items, namely management review, top-down communication, management commitment, resource allocation, and accountability $[3,10]$.

- Plan factor: This factor is associated with four items, namely quality policy, quality plan, reliable plan, and employee empowerment [13].

- Do factor: Five items are associated with this factor. They are documentation, quality awareness, rework, customer focus, and training $[12,8]$.

- Check factor: This factor consists of four items, including customer satisfaction, auditing, peer review, and competitiveness $[14,15]$. 
- Act factor: this factor is associated with four items: continuous improvement, preventive action, innovative action, and prompt response [9; 14].

\subsection{Importance Weights}

\subsubsection{Importance weight of key factors affecting quality performance}

The more relationships an item has on the other items, the more important it is. To be able to assess a construction quality performance, and progress through to higher levels of maturity, each of the five key factors is assigned with its importance weight. In this study, a total weight of 1,000 points is assigned based on the relationships a factor has on the other factors (see Figure 1). Leadership, Plan, Do, Check, and Act factors have the importance weights of 230, 256, 179, 230, and 105 points, respectively.



Fig. 1. Causal relationships of items affecting quality performance.

\subsubsection{Importance weight of items affecting quality performance}

The 22 items associated with five key factors also have different weight. This is based on the influences each item has on each other (see Figure 3). To explain, the "management commitment" item under the Leadership factor influences the other three items, namely the "top-down commitment", "quality policy", and "resource allocation" items. The "management review" item, on the other hand, has the influences on two items, which are the "quality policy" and "continuous improvement" items. The five items under the L eadership factor have a total of nine influences on the other items; this is converted to the importance weight of each item. For example, the "management commitment" item has the importance weight of $3 / 9$ or 0.33 of a maximum of 230 points (i.e. 75.9 points) of the Leadership factor. The importance weight of each item is summarized in Table 1.

\subsection{Influence Values among Key Factors Affecting Quality Performance}

Influences among key factors affecting quality performance are achieved from the number of influences, represented by a number of flows a factor has on another factor. Each influence value is calculated and normalized based on the total influences a factor has on the other 
factors, as shown in Table 2. The importance weights and influence values among the five key factors are later used for the dynamic model development.

Table 1. Importance Weight of the 22 Items Associated with Five Key Factors

\begin{tabular}{|c|c|c|c|}
\hline Factor & Item & Importance Weight & Maximum Score \\
\hline Leadership & M anagement commitment & 0.33 & 75.9 \\
& M anagement review & 0.22 & 50.6 \\
& Top-down communication & 0.22 & 50.6 \\
& Accountability & 0.12 & 27.6 \\
& Resources allocation & 0.11 & 25.3 \\
\hline Plan & Quality plan & 0.50 & 128.0 \\
& Employee empowerment & 0.30 & 76.8 \\
& Quality policy & 0.10 & 25.6 \\
& Unreliable plan & 0.10 & 25.6 \\
\hline Do & Customer focus & 0.44 & 79.0 \\
& Document information & 0.14 & 25.0 \\
& Quality awareness & 0.14 & 25.0 \\
& Rework & 0.14 & 25.0 \\
& Training & 0.14 & 25.0 \\
\hline Check & Peer review & 0.44 & 101.2 \\
& Customer satisfaction & 0.23 & 52.9 \\
& Auditing & 0.22 & 50.6 \\
& Competitiveness & 0.11 & 25.3 \\
\hline Act & Continuous improvement & 0.25 & 26.3 \\
& Preventive action & 0.25 & 26.3 \\
& Innovative action & 0.25 & 26.2 \\
& Prompt response & 0.25 & 26.2 \\
\hline & Total score & & 1000 \\
\hline
\end{tabular}

\section{Quality Performance Maturity Level}

The quality performance maturity level is developed in this study to assess the maturity level of construction quality performance. Each maturity level needs a score-range [16], for example, divided a total score of 1,000 points into five levels. Chinda [17], on the other hand, divided the 1,000 points into six safety maturity levels with the score-ranges. In this study, a total of 1000 points of quality performance are divided into five maturity levels based on the ISO 9001 and Plan-D o-Check-A ct Cycle. Level 1 of maturity has the score-range from 0 - 230 points, following the implementation of the Leadership factor. Level 2 of maturity then has the score-range from 231 - 486 points, implying the implementation of the Leadership and Plan factors. L evels 3, 4 and 5 have score-ranges of $487-665,666-$ 895 , and $896-1000$ points, respectively.

Table 2. Influence Values among Five Key Factors

\begin{tabular}{|c|c|c|c|c|c|}
\hline \multirow{2}{*}{ From } & \multicolumn{5}{|c|}{ To } \\
\cline { 2 - 6 } & Leadership & Plan & Do & Check & Act \\
\hline Leadership & - & 0.51 & 0.33 & - & 0.16 \\
\hline Plan & - & - & 0.50 & 0.25 & 0.25 \\
\hline Do & - & 0.33 & - & 0.67 & - \\
\hline Check & 0.25 & 0.13 & 0.37 & - & 0.25 \\
\hline Act & - & - & 0.67 & 0.33 & - \\
\hline
\end{tabular}


THEN (ROUND (Item1_D+item2_D+item3_D+item4_D+item5_D))

ELSE ((Item1_D+item2_D+item3_D+item4_D+item5_D), Max_Do)

\subsection{Check Factor Sub-model}

The total score of this factor is the summation of the four items' scores and the influential scores from the Plan, Do, and A ct factors (see Figure 5 and Table 2), see Equation 5.

Check score $=$ Min $($ IF $($ HISTORY (Item1_C,count_y)
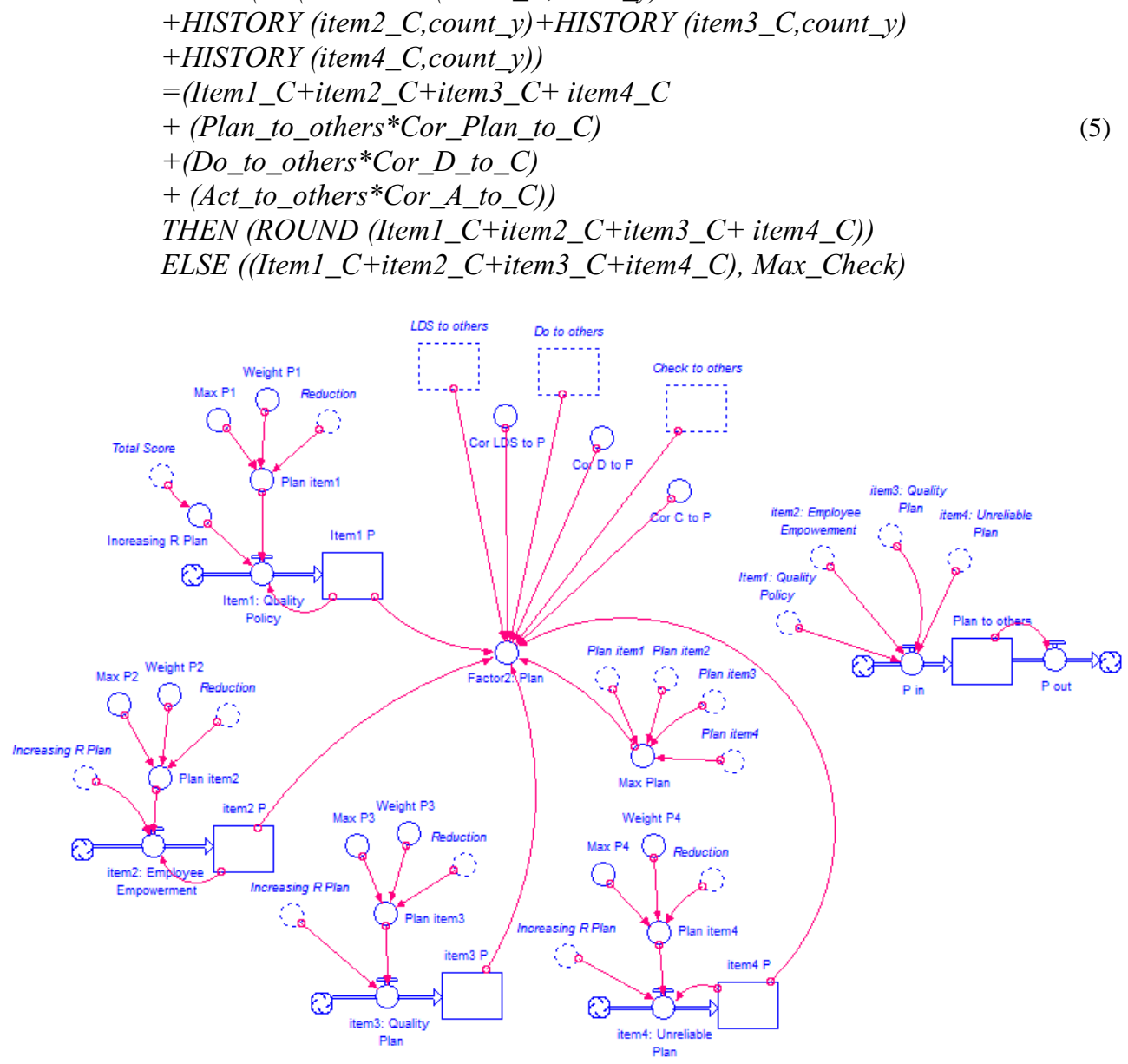

Fig. 2. Leadership Factor Sub-model. 




Fig. 3. Plan Factor Sub-model.

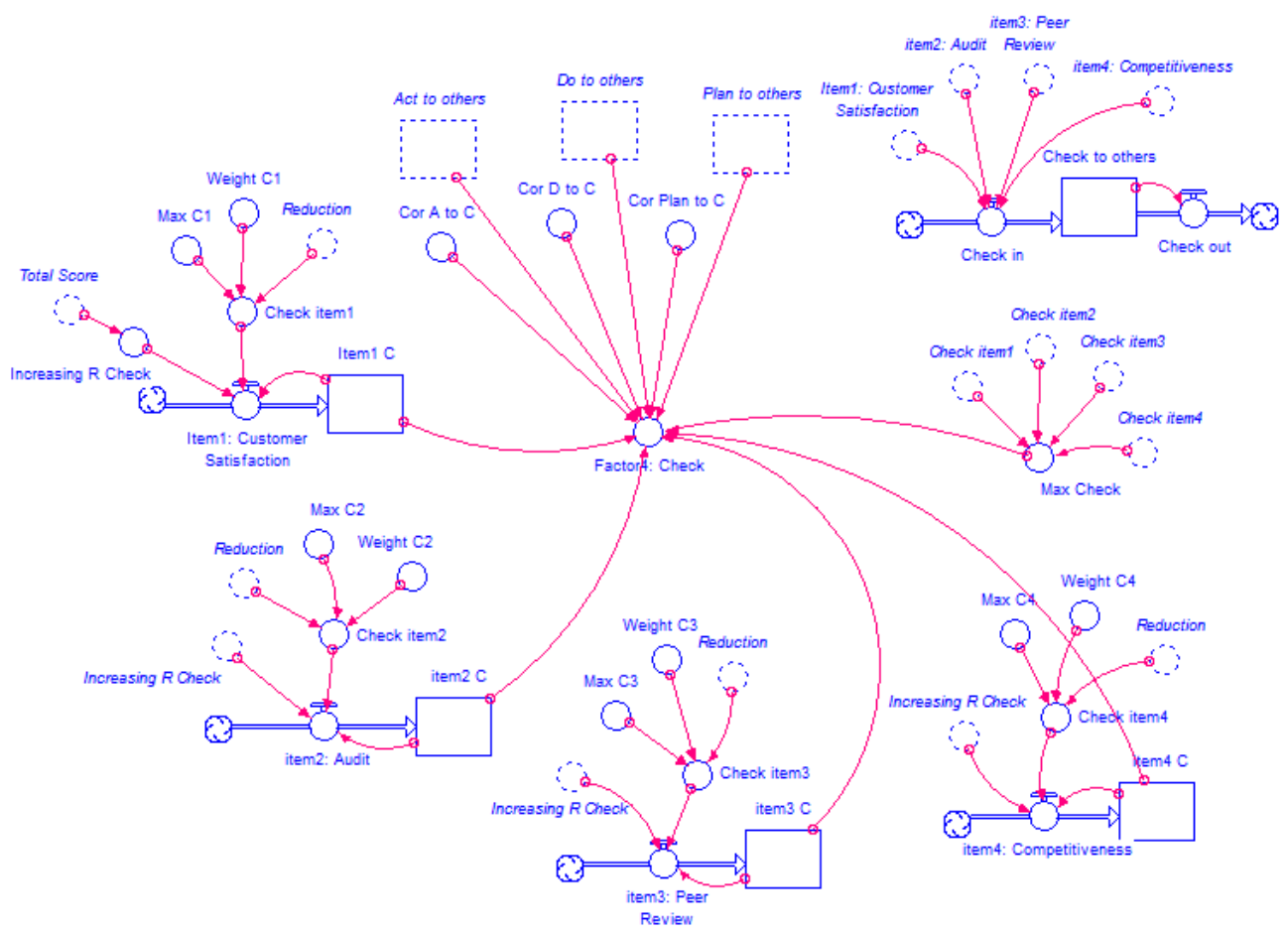

Fig. 4. Do Factor Sub-model. 


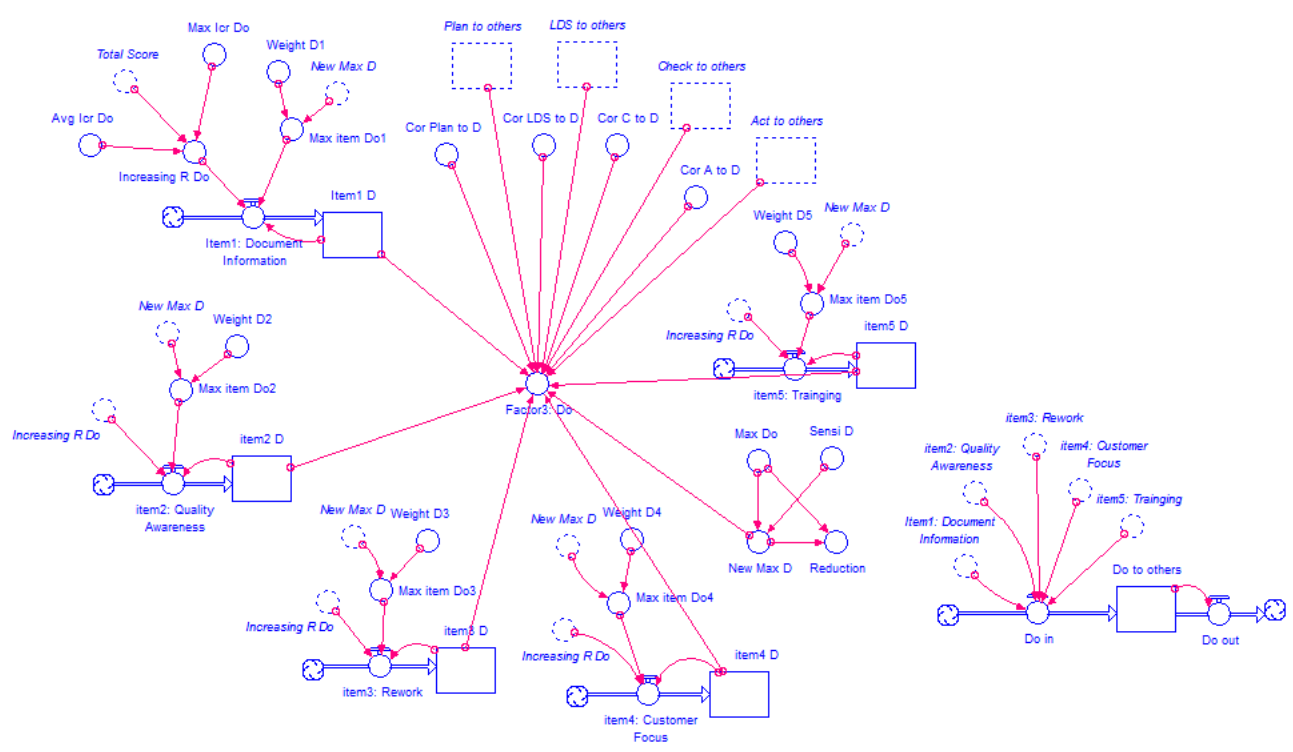

Fig. 5. Check Factor Sub-model.

\subsection{Act Factor Sub-model}

A ct factor sub-model is illustrated in Figure 6. The total score of this factor is the summation of the four items' scores and the influential scores from the Leadership, Plan, and Check factors (see Table 2), as shown in Equation 6. Based on the five sub-models, the total score of quality performance and the quality performance maturity level are calculated, see Equations 7 and 8.

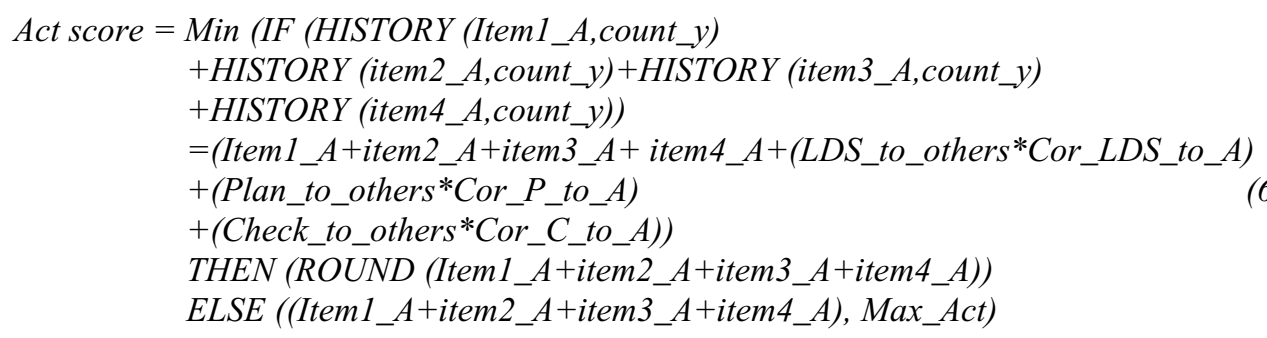

Total score $=$ Leadership score + Plan score

+ Do score+Check score+Act score

Maturity level $=I F((0 \leq$ Total_Score $)$ AND (Total_Score $\leq 230))$

THEN (1) ELSE (IF ((230<Total_Score) AND (Total_Score $\leq 486))$

THEN (2) ELSE (IF ( $(486<$ Total_Score) AND (Total_Score $\leq 665))$

THEN (3) ELSE (IF ((665<Total_Score) AND (Total_Score $\leq 895))$

THEN (4) ELSE (IF ( $(895<$ Total_Score) AND (Total_Score $\leq 1000)$

THEN (5) ELSE (0))))) 


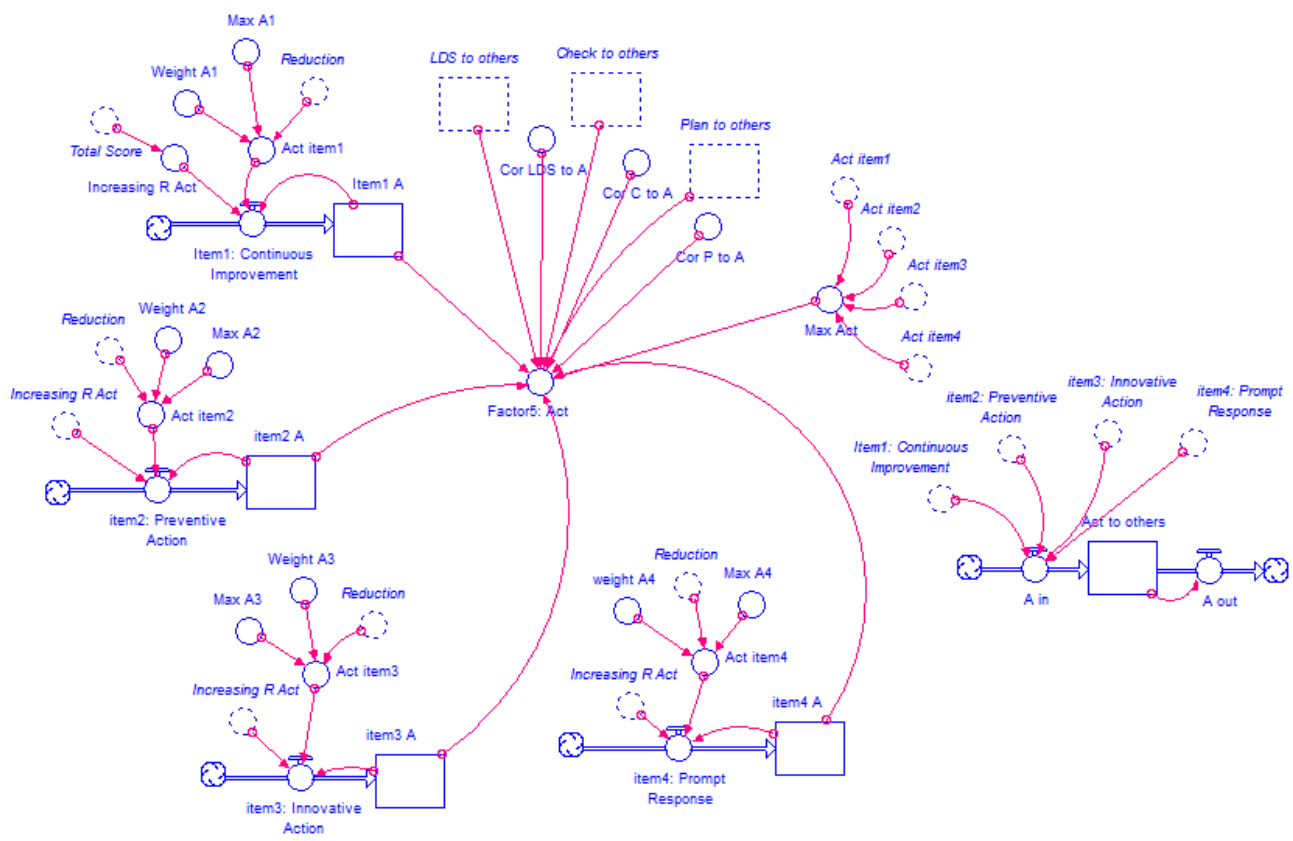

Fig. 6. Act Factor Sub-model.

\section{Simulation Results}

The dynamic model of construction quality performance is simulated, and the simulation results are as shown in Figure 7 . The results show that it takes six years for the company to implement the quality improvement program, and progress from level 1 to level 2 of maturity. W ith more experiences, the company spends four years each to achieve levels 2 and 3 of quality maturity. With cooperation from management and workers, the company reaches level 5 of quality performance maturity at the end of year 20. The results also show that the company achieves a perfect score of 1,000 points in 23 years.



Fig. 9. Graphical Results of Total Score and Maturity Level. 


\section{Conclusions}

W ith a growth in the country's economic, there is a need for Thai construction companies to achieve high-quality performance to gain competitive and conform to customer's requirement. This study utilizes the ISO 9001 and Plan-Do-Check-Act Cycle to develop a dynamic model of quality performance to examine the quality performance of the Thai construction industry in the long-term. The model consists of five sub-models, namely the Leadership, Plan, Do, Act, and Act sub-models. The simulation results reveal that the construction company struggles to progress to a higher level of maturity at the beginning years of quality improvement program implementation. This might be because of the lack of cooperation from management and working levels. W ith more management support and reliable quality policy, the company can achieve levels 3 and 4 in a shorter period of time. To progress through to level 5 of maturity, the continuous improvement plan must be executed. The results show that the company can achieve level 5 of quality performance maturity in 23 years. The dynamic model of quality performance developed in this study is based on the data gathered from construction-related literature. Primary data can be used to increase the accuracy of the model results. M oreover, each causal relationship is assumed to have equal importance. Further clarification could be performed using, for example, crossimpact analysis.

\section{References}

1. S.X. Zeng, P. Tian, J.J. Shi, Implementing integration of ISO 9001 and ISO 14001 for construction, M anagerial auditing journal, 20(4), 394-407, (2005)

2. J. Nolan, Would construction companies benefit fromISO9001? [online]. A vailable from: https://advisera.com/9001academy/blog/2016/06/07/would-constructioncompanies-benefit-from-iso-9001/ (2016)

3. A.A. Bubshait, T.H. AI-A tiq, ISO 9000 quality standards in construction, J ournal of management in engineering, 15(6), 41-46, (1999)

4. L.S. Pheng, J.A. Teo, Implementing total quality management in construction through ISO 9001: 2000. A rchitectural science review, 46(2), 159-165, (2003)

5. M. Chaudron, ISO 9001:2015 Key changes in the world's most popular QMS standard [online]. A vailable from: https://www.asqhouston.org (2016)

6. M. Gonzalez, The ISO 9001:2015sStandard and the Plan-Do-Check-Act Cycle [online]. A vailable from: https://www.qualitywbt.com/wp-content/uploads/TheISO-9001-2015-Standard-and-the-PDCA-Cycle.pdf (2016)

7. I. Paulova, M. Mlkva, Leadership - the key element in improving quality management, K valita inovacia prosperita/Quality innovation prosperity, 15(1), 2736, (2011)

8. S. Senaratne, J. Mayuran, Documentation management based on ISO for construction industries in developing countries, Journal of construction in developing countries, 20(2), 81-95, (2015)

9. B. N eyestani, Effectiveness of quality management system (QMS) on construction projects, M unich Personal RePEc A rchive, Department of Civil Engineering, De La Salle University, M anila, Philippines, (2016) 
10. H. Shah, J.R. Pitroda, A critical literature review on quality management for infrastructure projects, International journal of engineering research and development, 14(3), 18-21, (2018)

11. Management review [online]. A vailable from: https://www.iso-9001checklist.co.uk/tutorial/5.6-management-review.htm, ISO 9001, (2008)

12. N.M. Cachadinha, Implementing quality management systems in small and medium construction companies: a contribution to a road map for success, L eadership and management in engineering, 9(1), 32-39, (2009)

13. M. Hammar, Plan-Do-Check-Act in the ISO 9001 standard [online]. A vailable from: https://advisera.com/9001academy/knowledgebase/plan-do-check-act-inthe-iso-9001-standard/ (2018)

14. B. Neyestani, Impact of ISO 9001 certification on the projects' success of largescale $(A A A)$ construction firms in the Philippines. International research journal of management, IT and social sciences (IRJMIS), 3(11), 35-45, (2016)

15. N. Shaari, M.N. Abdullah, S.Z. Sheng, M.A.A. Lokman, Quality management system in contractor organizations: an empirical study on costs and benefits of ISO 9001: 2008 implementation. International journal of real estate studies, 11(5), 1-10, (2017)

16. Self-assessment: guidelines for Companies. European Foundation for Quality M anagement, B russels, B elgium, EFQM , 1998

17. T. Chinda, A safety assessment approach using safety enablers and results, International journal of occupational safety and ergonomics, 3, 343-361, (2012) 